Case Report

\title{
Upper Gastrointestinal Bleeding from Gastric Amyloidosis in a Patient with Smoldering Multiple Myeloma
}

\author{
Mihajlo Gjeorgjievski, ${ }^{1}$ Treta Purohit, ${ }^{2}$ Mitual B. Amin,,4 \\ Paul J. Kurtin, ${ }^{5}$ and Mitchell S. Cappel1 ${ }^{2,4}$ \\ ${ }^{1}$ Department of Internal Medicine, William Beaumont Hospital, Royal Oak, MI 48073, USA \\ ${ }^{2}$ Division of Gastroenterology \& Hepatology, William Beaumont Hospital, Royal Oak, MI 48073, USA \\ ${ }^{3}$ Anatomic Pathology, William Beaumont Hospital, Royal Oak, MI 48073, USA \\ ${ }^{4}$ Oakland University William Beaumont School of Medicine, Royal Oak, MI 48073, USA \\ ${ }^{5}$ Laboratory Medicine and Pathology, Mayo Clinic, Rochester, MN 55905, USA \\ Correspondence should be addressed to Mihajlo Gjeorgjievski; mihajlo.gjeorgjievski@beaumont.edu
}

Received 21 June 2015; Revised 29 July 2015; Accepted 29 July 2015

Academic Editor: Maria Teresa Bardella

Copyright (C) 2015 Mihajlo Gjeorgjievski et al. This is an open access article distributed under the Creative Commons Attribution License, which permits unrestricted use, distribution, and reproduction in any medium, provided the original work is properly cited.

\begin{abstract}
Amyloidosis is a common complication of patients with monoclonal gammopathy of undetermined significance (MGUS), smoldering multiple myeloma (SMM), and multiple myeloma (MM). This proteinaceous material can be deposited intercellularly in any organ system, including the gastrointestinal (GI) tract. In the GI tract, amyloidosis affects the duodenum most commonly, followed by the stomach and colorectum. Gastric amyloidosis causes symptoms of nausea, vomiting, early satiety, abdominal pain, and GI bleeding. A case of upper GI bleeding from gastric amyloidosis is presented in a patient with SMM. Esophagogastroduodenoscopy (EGD) revealed a gastric mass. Endoscopic biopsies revealed amyloid deposition in the lamina propria, consistent with gastric amyloidosis. Liquid chromatography tandem mass spectrometry performed on peptides extracted from Congo red-positive microdissected areas of paraffin-embedded stomach specimens revealed a peptide profile consistent with AL- (lambda-) type amyloidosis. Based on this and multiple other case reports, we recommend that patients with GI bleeding and MGUS, SMM, or MM undergo EGD and pathologic examination of endoscopic biopsies of identified lesions using Congo red stains for amyloidosis for early diagnosis and treatment.
\end{abstract}

\section{Introduction}

Patients with monoclonal gammopathy of undetermined significance (MGUS) and smoldering multiple myeloma (SMM) are at high risk to develop multiple myeloma (MM) and amyloidosis. The amyloidosis can infiltrate various organs, including any part of the gastrointestinal (GI) tract. Common symptoms of GI amyloidosis include GI bleeding, nausea, vomiting, and chronic abdominal pain [1-3]. Such GI symptoms in patients with known MGUS, SMM, or MM should prompt consideration of amyloidosis in the differential diagnosis, performance of GI endoscopy with biopsy, and special stains for amyloidosis on the endoscopic biopsies because the diagnosis of amyloidosis can alter the treatment and prognosis. A case is reported of GI bleeding associated with SMM, with GI amyloidosis diagnosed by esophagogastroduodenoscopy (EGD), Congo red stains performed on endoscopic biopsies, and liquid chromatography tandem mass spectrometry performed on peptides extracted after microdissection of Congo red-positive areas.

\section{Case Report}

A 92-year-old woman with a history of SMM presented with progressive weakness, lethargy, orthostatic dizziness, and melena during the prior few days. Bone marrow biopsy performed three months earlier had revealed a variably cellular marrow with trilineage hematopoiesis. The bone 


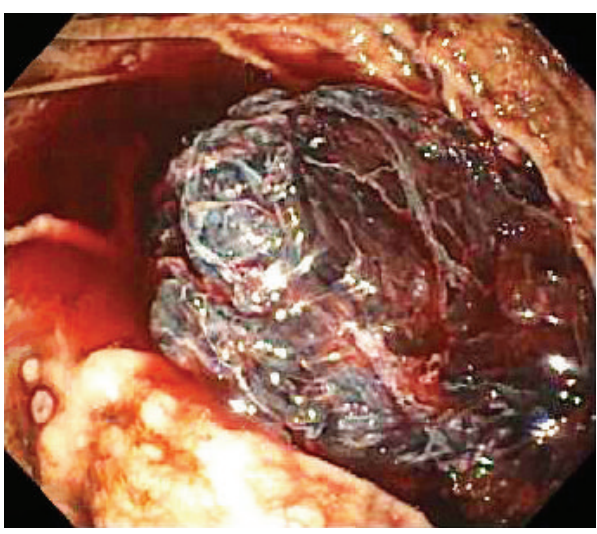

(a)

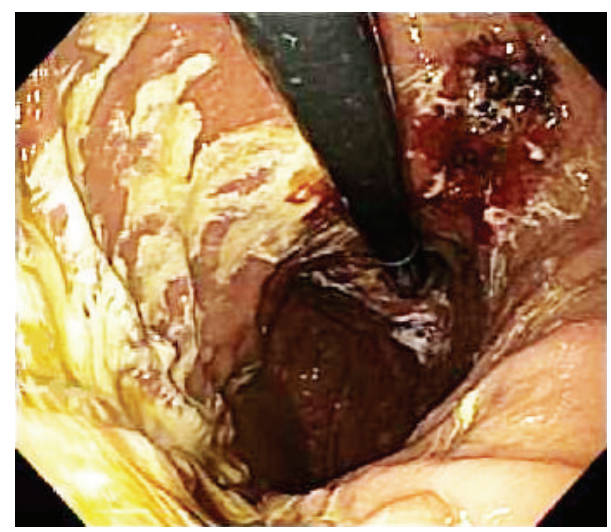

(b)

FIGURE 1: Initial EGD performed for an acute episode of melena in a 92-year-old woman with smoldering multiple myeloma (SMM) revealed a well-organized, hemispherical, $11 \times 6 \mathrm{~cm}$ tightly adherent clot attached to the midgastric body that that could not be detached despite vigorous endoscopic irrigation and aspiration (a). About eight, $3-5 \mathrm{~mm}$ wide sessile polyps (nodules) were present in the gastric fundus and body, devoid of stigmata of recent hemorrhage, two of which were present at the 8 oclock position. Repeat EGD performed one day later revealed that the clot had mostly dissolved exposing a $2.5 \times 2 \mathrm{~cm}$ ulcerated mass, mostly covered by a clot in the midgastric body along the lesser curvature (b).

marrow core biopsy and clot section contained numerous plasma cells, frequently in small clusters and in a few large aggregates, accounting for nearly $50 \%$ of the total cellularity. The plasma cells were small in size, were predominantly mature in morphology, and occasionally included atypical forms with slightly irregular nuclear contours and increased nuclear-to-cytoplasmic ratio. Immunohistochemical stains performed on the core biopsy revealed lambda light-chain restriction of CD138+ plasma cells and rare kappa+ plasma cells. A Congo red stain was not performed as part of the bone marrow biopsy. Serum immunoglobulin monoclonal protein (IgG lambda M-protein) was $2.2 \mathrm{mg} / \mathrm{dL}$. A 24-hour urine collection revealed $0.01 \mathrm{~g} / 24$ hours of albumin, with $0.07 \mathrm{~g} / 24$ hours of monoclonal free Lambda light chain (Bence-Jones proteins) as demonstrated by immunofixation. There was no evident end-organ disease, including absence of hypercalcemia, anemia, or renal failure. A complete bone survey revealed no lytic lesions.

Physical examination on admission revealed normal vital signs, a soft and nontender abdomen, and melena on rectal examination. The hematocrit was $32.5 \%$ (normal in females: $35.4 \%-44.2 \%)$. EGD revealed a large, hemispherical, $11 \times$ $6 \mathrm{~cm}$ adherent blood clot attached to the gastric body that could not be detached despite vigorous endoscopic irrigation and aspiration (Figure 1(a)). About eight small, sessile, polyps were present in the gastric fundus and body without stigmata of recent hemorrhage (SRH). No lesions were present in the esophagus or duodenum. The patient was administered intravenous pantoprazole at $8 \mathrm{mg} / \mathrm{hr}$ after an $80 \mathrm{mg}$ loading dose and transfused 2 units of packed erythrocytes. Repeat EGD performed one day later revealed that the clot had mostly dissolved exposing a not actively bleeding, $2.5 \times 2 \mathrm{~cm}$ ulcerated mass, mostly covered by clot in the midgastric body along the lesser curvature (Figure 1(b)). Histologic staining with Congo red stain revealed positively staining deposits in the lamina propria (Figure 2(a)) that exhibited apple-green birefringence under polarized light (Figures 2(b) and 2(c)), consistent with gastric amyloidosis. Peptides were extracted from Congo red-positive areas after microdissection of paraffin-embedded stomach biopsy specimens (Figure 3); and then liquid chromatography tandem mass spectrometry (LC MS/MS) was performed on the extracted peptides which revealed a peptide profile consistent with AL- (lambda-) type amyloidosis (Table 1).

Table 1 includes many of the proteins identified in the specimen as displayed by the Scaffold software. "Starred" entries are amyloid-associated proteins placed at the top of the list for ease of identification. Numbers marked by asterisks correspond to numbers in the mass spectrometry (MS) spectra for each identified protein. The asterisk over the spectral count represents the probability that the corresponding protein has been correctly identified. Spectral counts $\geq 5$ have $p>95 \%$. Spectral counts $<5$ are not interpreted because $p<95 \%$. Columns titled Patient Samples 1,2 , or 3 correspond to results from the 3 microdissections performed in Figure 3 (i.e., all cases analyzed by LC-MS/MS in triplicate). The samples contain abundant apolipoprotein A-IV, apolipoprotein E, and serum amyloid P-component peptides that are all codeposited in all types of amyloid. Additionally, all three replicate analyses contained abundant lambda light chain, which is the specific amyloid protein in this patient (i.e., the specimen contains amyloidosis, ALlambda light chain-type).

Other immunoglobulin proteins (gamma, alpha, and kappa) represent proteins in the background interstitial fluid/ serum. This finding is demonstrated by these proteins having much lower spectral counts than the lambda protein and by the presence in the sample of considerable albumin, a marker for "serum contamination." All the other listed proteins are typical tissue constituents, except for trypsin which was 


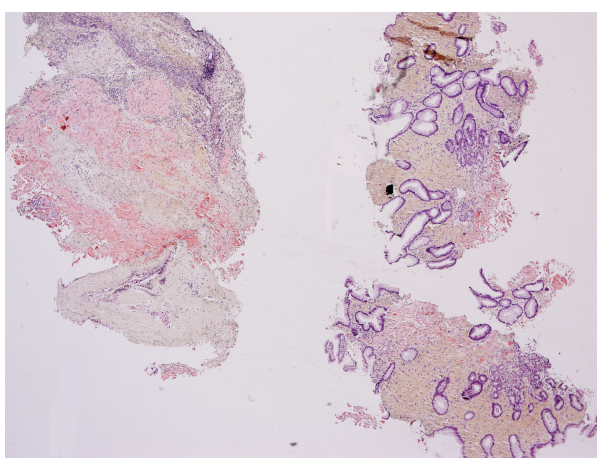

(a)

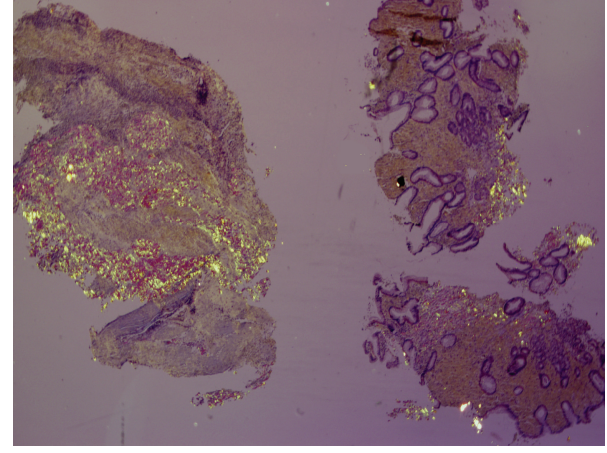

(b)

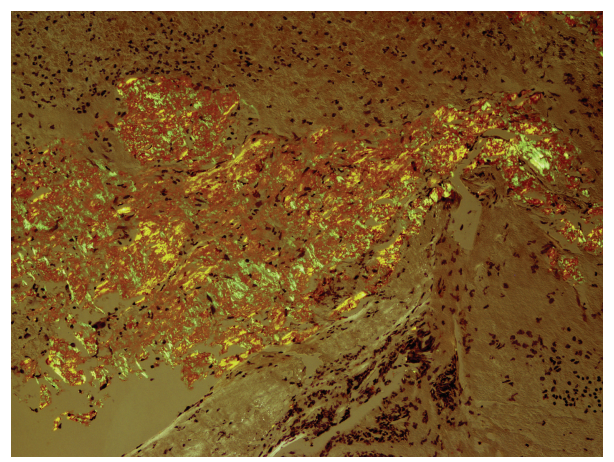

(c)

FiguRE 2: Low-power photomicrograph of endoscopic biopsies of the gastric mass using a Congo red stain revealed rose-pink staining of the amyloid deposits (a). Low-power (b) and high-power (c) photomicrographs of Congo red stain from the same sections using polarized light revealed the classic apple-green birefringence. Note that the regions exhibiting apple-green birefringence in (b) and (c) correspond to the same regions revealing rose-pink staining in (a). Note the presence of typical gastric glands on the right sides of (a) and (b).

added to the sample to digest the proteins prior to LCMS/MS analysis. The authors thank Jason D. Theis, B.S., in the Department of Laboratory Medicine and Pathology, at the Mayo Clinic, Rochester, Minnesota, for technical help in identifying the proteins in the specimen.

The patient had no further episodes of GI bleeding and was discharged home after 17 days in the hospital while receiving omeprazole $40 \mathrm{mg}$ orally once daily. No bleeding has been noted during one-month follow-up.

\section{Discussion}

SMM is an asymptomatic proliferative disorder of plasma cells that can progress to MM. It is differentiated from MM by absence of end-organ damage. SMM is far more likely to progress to MM or amyloidosis than MGUS (78\% versus $21 \%$ risk of progression) [4]. Amyloidosis is a condition of extracellular fibrillar protein deposition, that is, commonly associated with tissue injury and dysfunction [5]. Primary amyloidosis usually represents amyloidosis associated with immunocyte dyscrasia from monoclonal proliferation of plasma cells that synthesize an immunoglobulin that is prone to form amyloid.

GI amyloidosis can occur as an isolated entity or as part of multisystem involvement. Different types of amyloid proteins can deposit in various parts of the GI tract or liver, manifesting as abdominal pain, GI dysmotility, diarrhea, GI bleeding, or hepatic injury [1]. Suspected GI amyloidosis, irrespective of underlying etiology, requires biopsies of affected tissue [6]. GI involvement is uncommon in patients with amyloidosis, with a reported risk of only about 3\% [6]. Among patients with GI involvement, 79\% have underlying systemic amyloidosis, and $21 \%$ have only GI amyloidosis without evident plasma cell dyscrasia or extraintestinal involvement. Most systemic cases have underlying immunoglobulin light-chain disease (83\%) [6]. GI bleeding was the second most frequent symptom of amyloidosis (36\%), after weight loss (45\%), and closely followed by heartburn (33\%) [6]. Congo red stains are recommended for patients with unexplained weight loss, GI bleeding, abdominal pain, or early satiety associated with a monoclonal gammopathy [6]. In a case series of 37 cases of GI amyloidosis, the relative frequency of amyloid deposition was $100 \%$ in the duodenum, $95 \%$ in the stomach, and $91 \%$ in the colorectum [7].

In patients with primary systemic amyloidosis, Menke et al. [8] reported an $8 \%$ prevalence of GI involvement, with only $1 \%$ having symptomatic gastric involvement. Common presenting symptoms with gastric amyloidosis included hematemesis, prolonged nausea and vomiting, gastroparesis, or gastric outlet obstruction. Diagnosis of GI amyloidosis 
TABLE 1

\begin{tabular}{|c|c|c|c|c|c|c|}
\hline \# Starred & Bio View: identified proteins (649) & Accession number & Molecular weight & Patient sample 1 & $\begin{array}{c}\text { Patient } \\
\text { sample } 2\end{array}$ & Patient sample 3 \\
\hline $1 \hat{\swarrow}$ & Ig lambda-2 chain $\mathrm{C}$ regions & LAC2_HUMAN & $11 \mathrm{kDa}$ & $31^{*}$ & $21^{*}$ & $34^{*}$ \\
\hline $2 \widehat{\mho}$ & Apolipoprotein A-IV & APOA4_HUMAN & $45 \mathrm{kDa}$ & $28^{*}$ & $17^{*}$ & $15^{*}$ \\
\hline $3 \hat{\omega}$ & Apolipoprotein E & APOE_HUMAN & $36 \mathrm{kDa}$ & $21^{*}$ & $11^{*}$ & $15^{*}$ \\
\hline$\hat{\omega}$ & Serum amyloid P-component & SAMP_HUMAN & $25 \mathrm{kDa}$ & $22^{*}$ & $13^{*}$ & $13^{*}$ \\
\hline$\hat{\omega}$ & Ig gamma-1 chain $\mathrm{C}$ region & IGHG1_HUMAN & $36 \mathrm{kDa}$ & $6^{*}$ & $10^{*}$ & $7^{*}$ \\
\hline$\hat{\omega}$ & Ig alpha-1 chain $\mathrm{C}$ region & IGHA1_ HUMAN & $38 \mathrm{kDa}$ & $6^{*}$ & $5^{*}$ & \\
\hline$\hat{\omega}$ & Ig gamma-3 chain $\mathrm{C}$ region & IGHG3_HUMAN & $41 \mathrm{kDa}$ & $4^{*}$ & $7^{*}$ & \\
\hline$\hat{\omega}$ & Ig kappa chain $\mathrm{C}$ region & IGKC_HUMAN & $12 \mathrm{kDa}$ & $4^{*}$ & $3^{*}$ & \\
\hline$\hat{\omega}$ & Ig kappa chain V-III region... & KV302_HUMAN... & $12 \mathrm{kDa}$ & $4^{*}$ & & \\
\hline $10 \widehat{\downarrow}$ & Ig lambda chain V-II region... & LV205_HUMAN & $12 \mathrm{kDa}$ & & $3^{*}$ & \\
\hline 11 & (ENZYME) trypsin precursor & ENZYME_TRYP_... & $24 \mathrm{kDa}$ & $230^{*}$ & $223^{*}$ & $220^{*}$ \\
\hline 12 & Vitronectin & VTNC_HUMAN & $54 \mathrm{kDa}$ & $38^{*}$ & $31^{*}$ & $40^{*}$ \\
\hline 13 & Keratin, type II cytoskeletal... & K2C1_HUMAN & $66 \mathrm{kDa}$ & $6^{*}$ & $49^{*}$ & $20^{*}$ \\
\hline 14 & Keratin, type I cytoskeletal. .. & K1C10_HUMAN & $59 \mathrm{kDa}$ & $7^{*}$ & $35^{*}$ & $12^{*}$ \\
\hline 15 & Apolipoprotein A-I & APOA1_HUMAN & $31 \mathrm{kDa}$ & $20^{*}$ & $20^{*}$ & $5^{*}$ \\
\hline 16 & Serum albumin & ALBU_HUMAN & $69 \mathrm{kDa}$ & $8^{*}$ & $15^{*}$ & $15^{*}$ \\
\hline 17 & Keratin, type II cytoskeletal... & K22E_HUMAN & $65 \mathrm{kDa}$ & & $33^{*}$ & $1^{* *}$ \\
\hline 18 & Hemoglobin subunit alpha & HBA_HUMAN & $15 \mathrm{kDa}$ & $6^{*}$ & $3^{* *}$ & $24^{*}$ \\
\hline 19 & Complement component C9 & CO9_HUMAN & $63 \mathrm{kDa}$ & $16^{*}$ & $16^{*}$ & \\
\hline 20 & Keratin, type I cytoskeletal 9 & K1C9_HUMAN & $62 \mathrm{kDa}$ & & $21^{*}$ & $10^{*}$ \\
\hline 21 & Hemoglobin subunit beta & HBB_HUMAN & $16 \mathrm{kDa}$ & $6^{*}$ & $2^{*}$ & $20^{*}$ \\
\hline 22 & Plasminogen & PLMN_HUMAN & $91 \mathrm{kDa}$ & $15^{*}$ & $13^{*}$ & $1^{* *}$ \\
\hline 23 & Fibrinogen alpha chain & FIBA_HUMAN & $95 \mathrm{kDa}$ & & & $31^{*}$ \\
\hline 24 & Fibrinogen beta chain & FIBB_HUMAN & $56 \mathrm{kDa}$ & & & $27^{*}$ \\
\hline 25 & Fibrinogen gamma chain & FIBG_HUMAN & $52 \mathrm{kDa}$ & & & $25^{*}$ \\
\hline 26 & Collagen alpha-1(I) chain & CO1A1_HUMAN & $139 \mathrm{kDa}$ & $6^{*}$ & $1^{* *}$ & $12^{*}$ \\
\hline 27 & Collagen alpha-2(I) chain & CO1A2_HUMAN & $129 \mathrm{kDa}$ & $11^{*}$ & & $8^{*}$ \\
\hline 28 & Trypsin-3 & TRY3_HUMAN & $33 \mathrm{kDa}$ & $4^{*}$ & $4^{*}$ & $4^{*}$ \\
\hline 29 & Collagen alpha-3(VI) chain & CO6A3_HUMAN & $344 \mathrm{kDa}$ & $2^{*}$ & $6^{*}$ & $8^{*}$ \\
\hline 30 & 395 ribosomal protein $L 40, \ldots$ & RM40_HUMAN & $24 \mathrm{kDa}$ & $3^{* *}$ & $2^{* *}$ & $4^{* *}$ \\
\hline
\end{tabular}

Probability legend: ${ }^{*}$ over $95 \%$; ${ }^{* *} 80 \%$ to $94 \%$.

without previously diagnosed inflammatory or plasma cell disorders is exceptionally rare [9]. In one case report, it has been proposed that amyloidosis should always be considered in the differential diagnosis of hematemesis and gastric tumors [10]. In another case report, a strong suspicion of amyloidosis was recommended in patients with multiple myeloma and obscure GI bleeding [2].

The endoscopic appearance of gastric amyloidosis can closely resemble that of gastric malignancy [10]. Amyloidosis can appear as submucosal tumors, polyps, antral narrowing, thickened irregular gastric folds, or loss of rugal folds. Other appearances include gastric ulcers, hematomas, arteriovenous malformations, granular-appearing mucosa, plaquelike lesions, or ulcerative gastritis, often associated with GI bleeding [3].

The pathophysiology of GI bleeding from amyloidosis involves local ischemia, infarction, and mucosal injury that cause erosions, hematomas, or ulcerations. When GI bleeding 


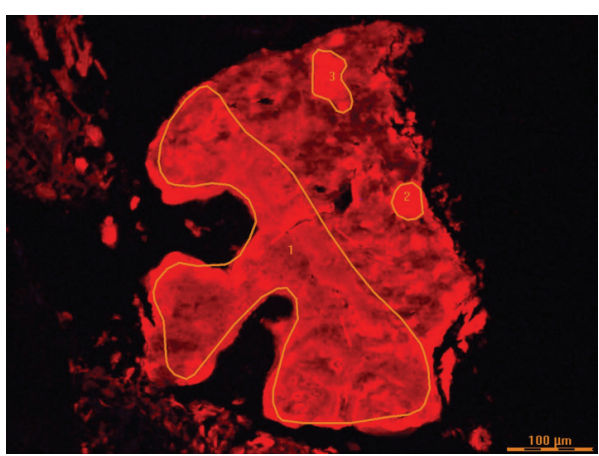

(a)

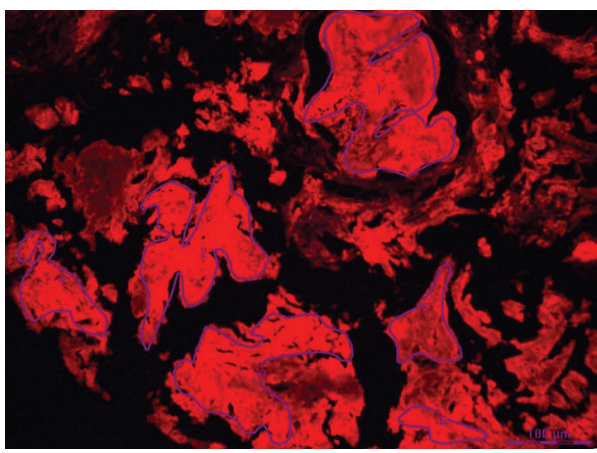

(c)

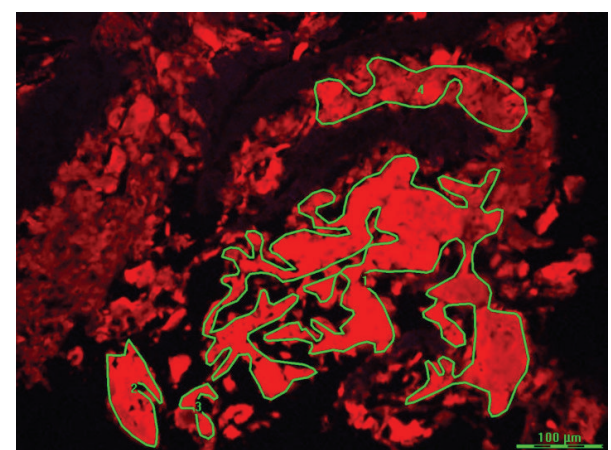

(b)

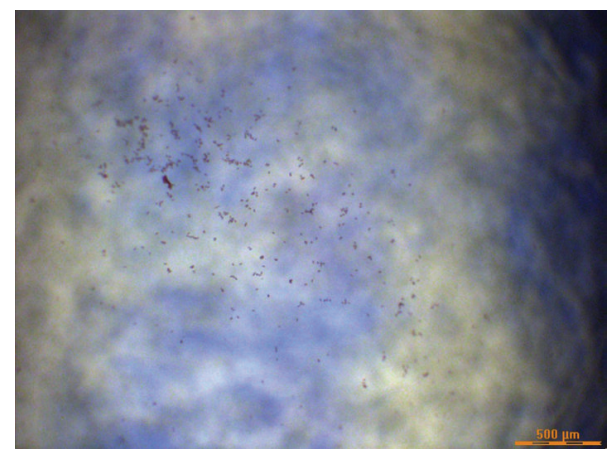

(d)

Figure 3: Congo red-positive sections viewed under ultraviolet light in the microdissection microscope. The amyloid fluoresces red. The drawn lines (yellow in (a), green in (b), and blue in (c)) delineate areas circumscribing the amyloid deposits that were cut out of the tissue sections for processing for mass spectrometry. The three dissections in (a)-(c) correspond to the three patient sample columns in the Scaffold illustration in Table 1. Each sample was processed in triplicate. (d) shows the amyloid fragments that have been cut out of the tissue and are now in the reaction cup for further processing (i.e., reduction of disulfide bonds, trypsin digestion, etc.) before mass spectrometry.

is the presenting symptom, endoscopy commonly reveals a submucosal hematoma [7, 11]. GI bleeding caused by gastric amyloidosis may be obscure or overt. Hematoma rupture in patients with gastric amyloidosis can sometimes cause life-threatening, GI bleeding. Currently, no treatment guidelines exist for endoscopic therapy for bleeding from gastric amyloidosis, and the decision on which endoscopic therapy is used is based on the type of lesion present and the endoscopist's preference. Endoscopic hemostasis is often ineffective and surgical intervention may be required [12]. In the currently reported patient endoscopic therapy was not performed because there was no active source of bleeding during repeat EGD. The patient was placed on intravenous proton pump inhibitor initially which was later switched to omeprazole $40 \mathrm{mg}$ twice a day. There was no recurrent bleeding during one month of follow-up.

\section{Conflict of Interests}

This paper does not discuss any confidential pharmaceutical industry data reviewed by Dr. Cappell as a consultant for the United States Food \& Drug Administration (FDA) Advisory Committee on Gastrointestinal Drugs. Dr. Cappell is a member of the speaker's bureau of AstraZeneca. This work does not discuss any drug manufactured or marketed by AstraZeneca. All authors declare no conflict of interests.

\section{Authors' Contribution}

Dr. Mihajlo Gjeorgjievski and Dr. Mitchell S. Cappell contributed equally to this paper.

\section{References}

[1] S. Petre, I. A. Shah, and N. Gilani, "Review article: gastrointestinal amyloidosis: clinical features, diagnosis and therapy," Alimentary Pharmacology and Therapeutics, vol. 27, no. 11, pp. 1006-1016, 2008.

[2] S.-S. Chang, C.-L. Lu, S.-H. Tsay, F.-Y. Chang, and S.-D. Lee, "Amyloidosis-induced gastrointestinal bleeding in a patient with multiple myeloma," Journal of Clinical Gastroenterology, vol. 32, no. 2, pp. 161-163, 2001.

[3] E. C. Ebert and M. Nagar, "Gastrointestinal manifestations of amyloidosis," The American Journal of Gastroenterology, vol. 103, no. 3, pp. 776-787, 2008.

[4] R. A. Kyle, E. D. Remstein, T. M. Therneau et al., "Clinical course and prognosis of smoldering (asymptomatic) multiple myeloma," The New England Journal of Medicine, vol. 356, no. 25, pp. 2582-2590, 2007. 
[5] J. D. Gillmore and P. N. Hawkins, "Pathophysiology and treatment of systemic amyloidosis," Nature Reviews Nephrology, vol. 9, no. 10, pp. 574-586, 2013.

[6] A. J. Cowan, M. Skinner, D. C. Seldin et al., "Amyloidosis of the gastrointestinal tract: a 13-year, single-center, referral experience," Haematologica, vol. 98, no. 1, pp. 141-146, 2013.

[7] S. Tada, M. Iida, A. Iwashita et al., "Endoscopic and biopsy findings of the upper digestive tract in patients with amyloidosis," Gastrointestinal Endoscopy, vol. 36, no. 1, pp. 10-14, 1990.

[8] D. M. Menke, R. A. Kyle, C. R. Fleming, J. T. Wolfe III, P. J. Kurtin, and W. A. Oldenburg, "Symptomatic gastric amyloidosis in patients with primary systemic amyloidosis," Mayo Clinic Proceedings, vol. 68, no. 8, pp. 763-767, 1993.

[9] R. Fossmark, E. Skarsvåg, H. Aarset, H. Hjorth-Hansen, and H. L. Waldum, "Symptomatic primary (Al) amyloidosis of the stomach and duodenum," Case Reports in Gastrointestinal Medicine, vol. 2013, Article ID 525439, 3 pages, 2013.

[10] S. Björnsson, J. H. Jóhannsson, and F. Sigurjónsson, “Localized primary amyloidosis of the stomach presenting with gastric hemorrhage," Acta Medica Scandinavica, vol. 221, no. 1, pp. 115119, 1987.

[11] D. G. James, G. R. Zuckerman, G. S. Sayuk, H. L. Wang, and C. Prakash, "Clinical recognition of Al type amyloidosis of the luminal gastrointestinal tract," Clinical Gastroenterology and Hepatology, vol. 5, no. 5, pp. 582-588, 2007.

[12] Y.-C. Yeh, C.-H. Lin, S.-C. Huang, and Y.-K. Tsou, "Gastrointestinal: gastric hematoma with bleeding in a patient with primary amyloidosis," Journal of Gastroenterology and Hepatology (Australia), vol. 29, no. 3, pp. 419-419, 2014. 


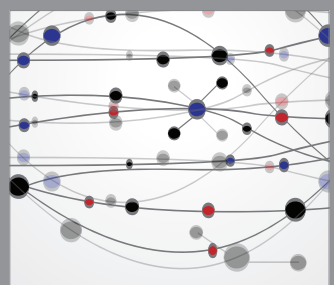

The Scientific World Journal
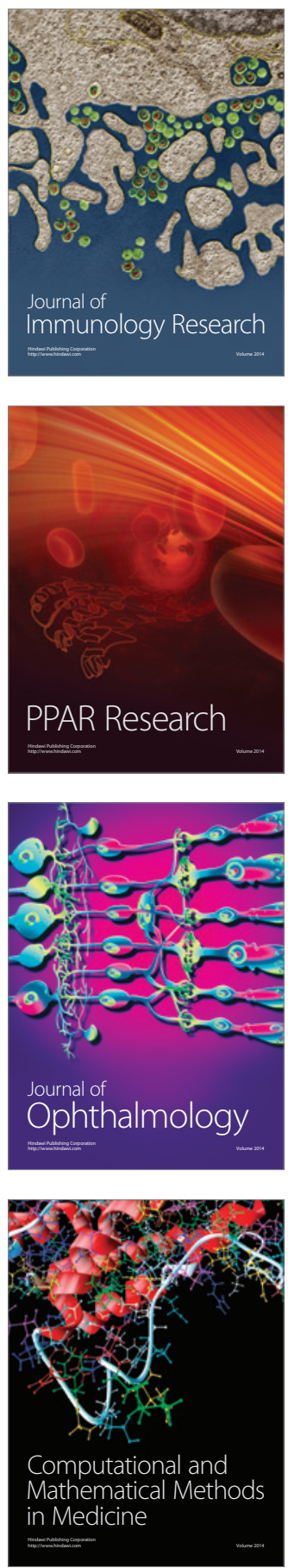

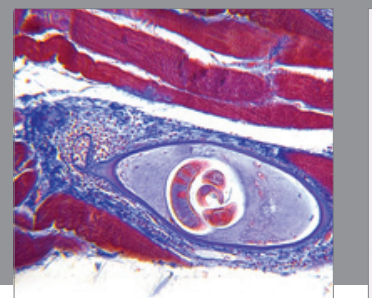

Gastroenterology

Research and Practice
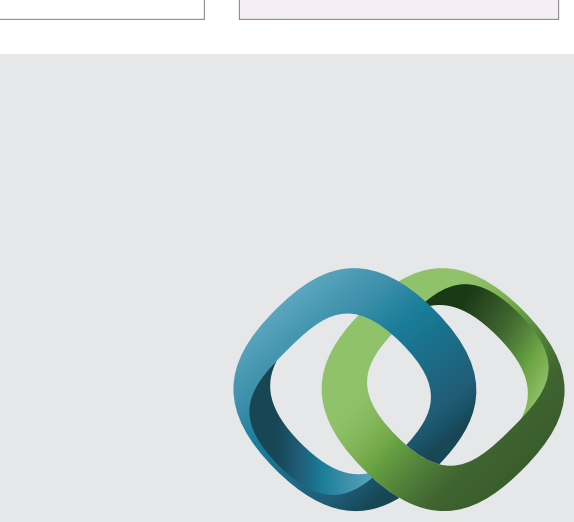

\section{Hindawi}

Submit your manuscripts at

http://www.hindawi.com
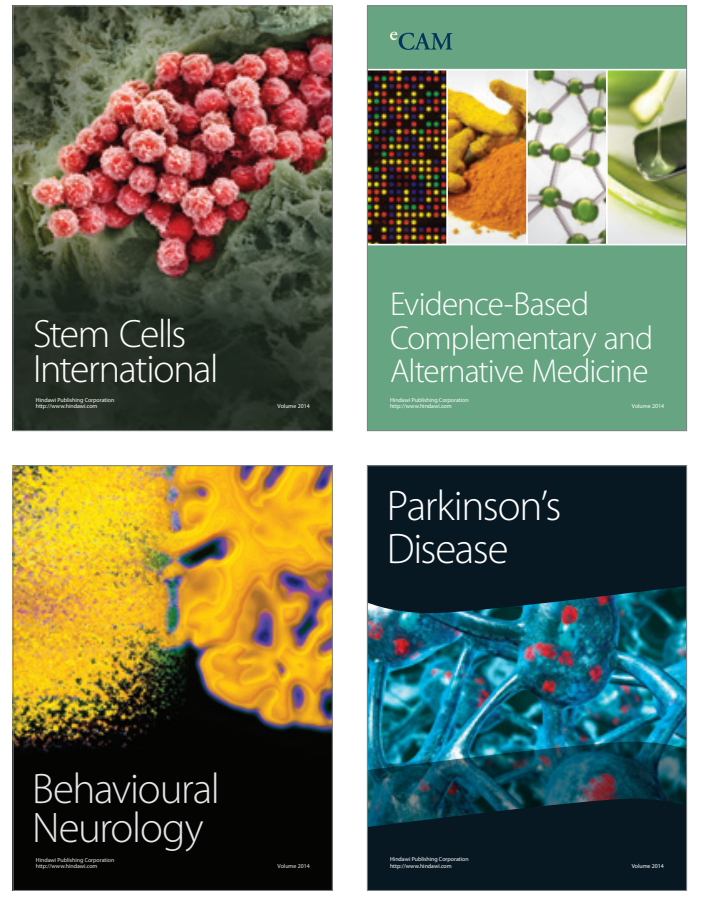
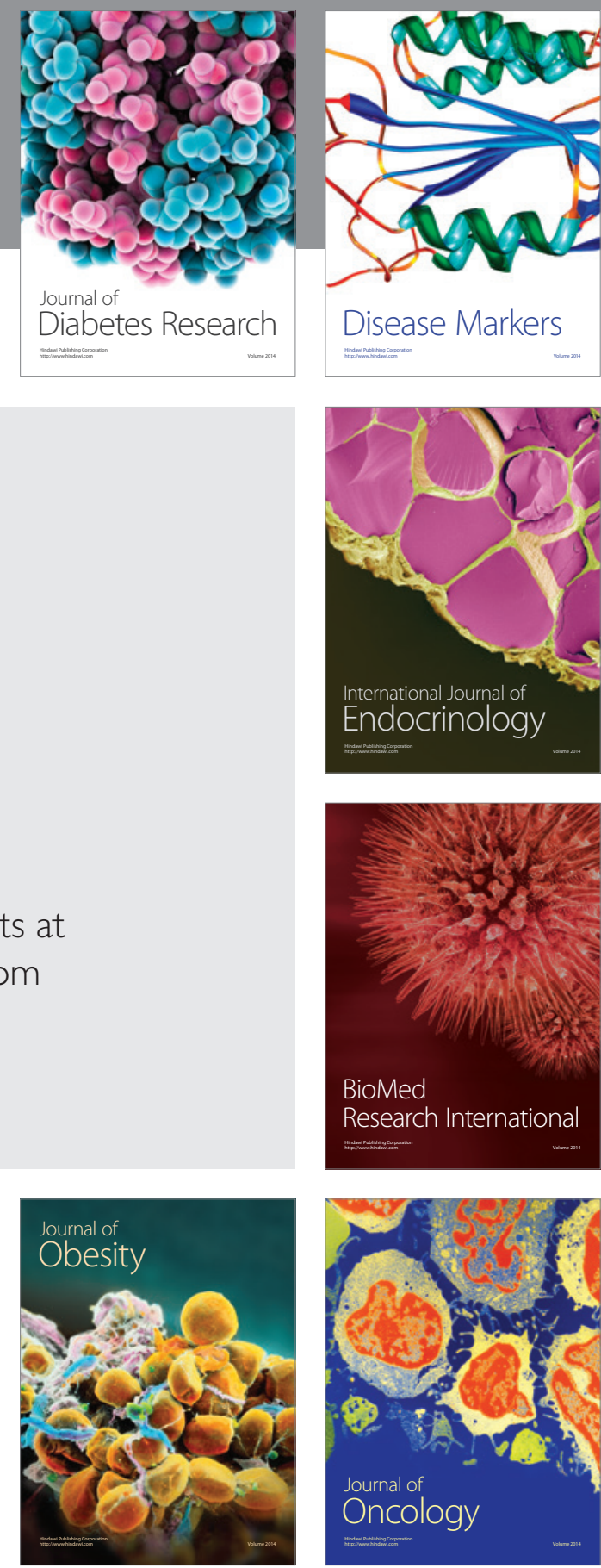

Disease Markers
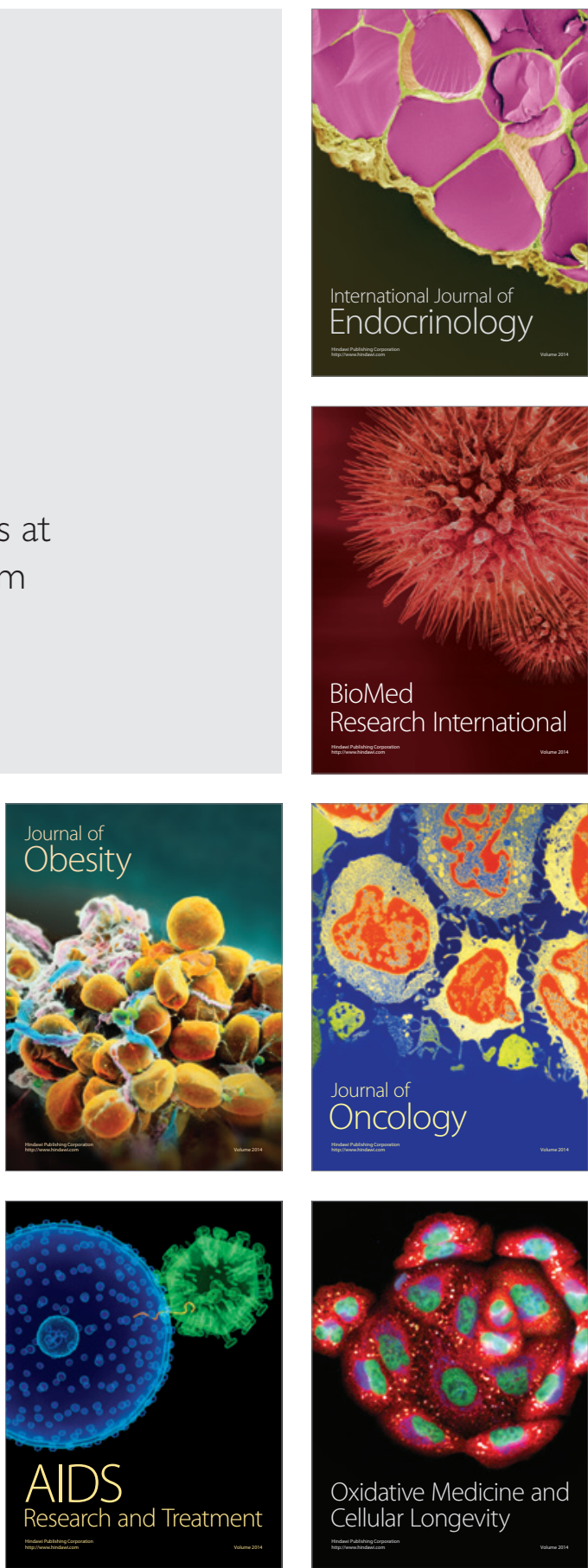